\title{
A mid-infrared exploration of the dusty environments of active galactic nuclei
}

\author{
Almudena Alonso-Herrero ${ }^{1,2}$ \\ ${ }^{1}$ Instituto de Física de Cantabria, CSIC-UC, 39005 Santander, Spain \\ email: aalonso@ifca.unican.es \\ ${ }^{2}$ Augusto G. Linares Senior Research Fellow
}

\begin{abstract}
We present the first results from a mid-infrared survey of local Active Galactic Nuclei (AGN) using the CanariCam (CC) instrument on the $10.4 \mathrm{~m}$ Gran Telescopio Canarias (GTC). We are obtaining sub-arcsecond angular resolution $(0.3-0.6$ arcsec) mid-IR imaging and spectroscopic observations of a sample of 100 local AGN, which are complemented with data taken with T-ReCS, VISIR, and Michelle. The full sample contains approximately 140 AGN, covers nearly six orders of magnitude in AGN luminosity, and includes low-luminosity AGN (LLAGN), Seyfert 1s and 2s, QSO, radio galaxies, and (U)LIRGs. The main goals of this project are: (1) to test whether the properties of the dusty tori of the AGN Unified Model depend on the AGN type, (2) to study the nuclear star formation activity and obscuration of local AGN, and (3) to explore the role of the dusty torus in LLAGN.
\end{abstract}

Keywords. Active Galactic Nuclei, Infrared, Star Formation

\section{Introduction}

In the context of the Unified Model (Antonucci 1993) for active galactic nuclei (AGN), it is generally accepted that the obscuring material is located in an optically thick dusty torus. From the modelling of nuclear infrared (IR) observations it has become clear that AGN tori are relatively compact (from a few parsecs to a few tens of parsecs). Moreover, the torus models reproduce better the observations if the dust is mostly distributed in clumps rather than homogeneously (see e.g., Tristram et al. 2009, Ramos Almeida et al. 2009, 2011, Alonso-Herrero et al. 2011). However, it is still an open question whether the properties of the torus vary with AGN type and/or the luminosity of the AGN. In addition, the tori are not isolated structures in the nuclear regions of AGN. Rather, IR observations have shown that there is some continuity between the dust in the torus and that in the nuclear environments (Roche et al. 2006, Packham et al. 2007). Finally, models also predict that star formation (SF) should occur inside or near the outer edge of the dusty tori (see e.g., Wada \& Norman 2002).

The dust in the torus absorbs a large fraction of the AGN emission and re-emits it in the IR peaking at mid-IR $(\sim 8-40 \mu \mathrm{m})$ wavelengths. Therefore, sub-arcsecond mid-IR observations offer the best opportunity to constrain the torus models and learn about the dusty environments of AGN. The angular resolution achieved with ground-based 8-10 m class telescopes, although it is not sufficiently high to spatially resolve the torus structure, can help disentangle the torus emission from the circumnuclear and host galaxy contribution (see e.g., Packham et al. 2005, Roche et al. 2006). We present results from a mid-IR survey of a sample of 100 local AGN using the CanariCam (CC, Telesco et al. 2003) instrument on the $10.4 \mathrm{~m}$ Gran Telescopio Canarias (GTC). The main goal of this project is to test whether the properties of the torus depend on the AGN type and 

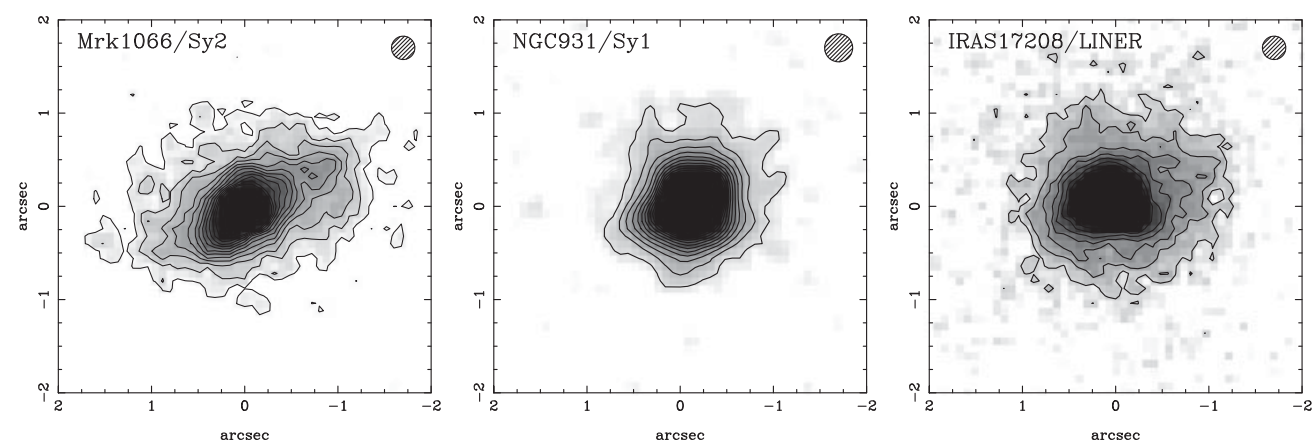

Figure 1. GTC/CC $8.7 \mu \mathrm{m}$ images of three AGN in our sample. The hatched circles at the top right of the panels are the resolution of the images (FWHM).

luminosity and explore the environments of the dusty torus by studying the nuclear star formation $(\mathrm{SF})$ and extinction produced by the host galaxy†.

\section{High angular resolution ground-based mid-IR observations}

The observations are part of the CC AGN guaranteed time and from an ESO/GTC large programme (182.B-2005) for a total of 280 hours. The observations include highangular resolution $(0.3-0.6$ arcsec $)$ imaging with the Si-2 $\left(\lambda_{\mathrm{c}}=8.7 \mu \mathrm{m}\right)$ filter and lowresolution (nominal $R=\lambda / \Delta \lambda \simeq 175$ ) spectroscopy covering the $\sim 8-13 \mu \mathrm{m}$ spectral range. We complemented the CC data with existing observations with the T-ReCS, Michelle, and VISIR instruments for approximately 40 Seyfert and LLAGN. The full sample covers more than six decades of AGN luminosity and includes LLAGN, Seyfert $1 \mathrm{~s}$ and $2 \mathrm{~s}$, radio galaxies, quasars and IR luminous and ultraluminous IR galaxies (LIRGs and ULIRGs) hosting AGN. Fig. 1 shows the GTC/CC $8.7 \mu \mathrm{m}$ images of three AGN in our sample reduced with the CC pipeline REDCAN (González-Martín et al. 2013).

\section{Results}

\subsection{Torus properties of Seyfert galaxies}

Modelling the nuclear IR emission of nearby AGN with torus models can be used to derive torus properties such as its geometry, distribution and optical depth of the dust in the torus, and the AGN luminosity and covering factor. If this is done for a large sample of AGN, it is then possible to investigate whether such properties depend on the AGN type (Type 1 vs. Type 2), AGN class (AGN in LIRGs and ULIRGs, radio galaxies, Seyferts), and AGN luminosity. Over the past few years we fitted sub-arcsecond angular resolution IR spectral energy distributions (SEDs) and mid-IR spectroscopy of relatively small samples of Seyferts and individual objects (see e.g., Ramos Almeida et al. 2009, 2011, Alonso-Herrero et al. 2011, 2013) using Nenkova (2008) clumpy torus models (also known as CLUMPY). To deal with the intrinsic degeneracies of the CLUMPY models, we used the Bayesian fitting tool BAYEsclumpy (Asensio Ramos \& Ramos Almeida 2009), which allows us to derive probability distributions of the fitted parameters (e.g., torus extent and angular size, number and distribution of the clouds).

Fig. 2 (left) shows the best fit to the IR SED and the GTC/CC mid-IR spectrum of the nuclear region of NGC 3690, one of the components of the interacting LIRG Arp 299. The high angular resolution $(0.3 \mathrm{arcsec})$ of the $\mathrm{CC}$ data allowed us to probe nuclear physical scales of approximately $60 \mathrm{pc}$, which is a factor of 10 improvement over previous mid-IR

$\dagger$ Additional information about this project can be found at: https://sites.google.com/ site/piratasrelatedpublications/ 

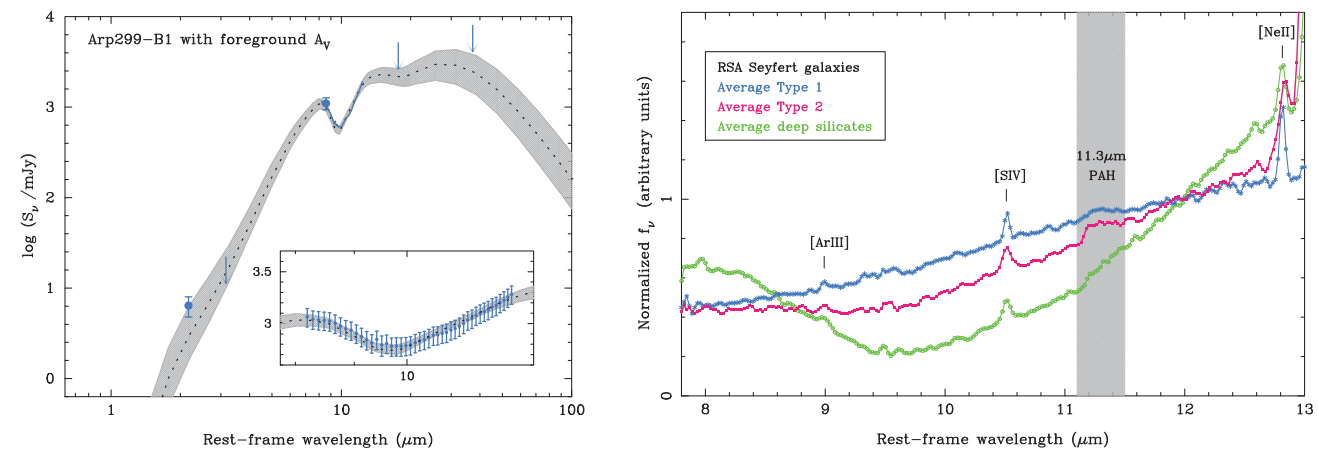

Figure 2. Left panel. Fit to the IR SED (filled symbols and upper limits) and GTC/CC nuclear spectrum (solid line) of NGC 3690/Arp 299-B1 using the CLUMPY models (dotted line and shaded area) of Nenkova et al. (2008). Adapted from Alonso-Herrero et al. (2013). Right panel. Average nuclear spectra normalized at $12 \mu \mathrm{m}$ of the Seyfert 1s (asterisks), Seyfert 2s (filled squares), and Seyfert nuclei with deep $10 \mu \mathrm{m}$ silicate features (open dots) in the RSA sample studied by Esquej et al. (2013). We mark the most important spectral features including the $11.3 \mu \mathrm{m}$ PAH feature that is used to probe SF on nuclear scales (see Section 3.3).

spectroscopy of this system. From the fit done using the Nenkova et al. (2008) torus models we inferred that the IR emission of this nucleus comes from AGN-heated dust in a clumpy torus with both a high covering factor and high extinction along the line of sight (see Alonso-Herrero et al. 2013 for more details).

Although so far we studied the torus properties for relatively small AGN samples, our recent work indicates some dependency of the torus geometry, in particular the covering factor, on the Type1/Type2 class (Ramos Almeida et al. 2011) and/or the luminosity of the AGN (Alonso-Herrero et al. 2011). These works also highlighted the importance of disentangling the dust heated by the AGN from foreground dust in the host galaxy when modelling the nuclear IR emission of local AGN (see also, González-Martín et al. 2013).

\subsection{Nuclear Star Formation Activity in Seyfert galaxies}

Since material must be driven inwards from the interstellar medium of the host galaxy to the nucleus ( $<1$ pc regions) to fuel the AGN, nuclear SF on scales of less than $\sim 100 \mathrm{pc}$ appears to be an inevitable consequence of this process, as predicted by numerical simulation (see e.g., Hopkins \& Quataert 2010). We can use the mid-IR polycyclic aromatic hydrocarbon (PAH) features to probe the nuclear SF activity in local AGN. In particular, it has been shown that the $11.3 \mu \mathrm{m}$ PAH feature is not suppressed due to the presence of an AGN on kiloparsec scales (Diamond-Stanic \& Rieke 2010) down to distances from the AGN of up to a few tens of parsecs (Esquej et al. 2013).

Esquej et al. (2013) compiled mid-IR nuclear spectra obtained with T-ReCS, Michelle, and VISIR at sub-arcsecond angular resolutions for 29 Seyfert galaxies in the revised Shapley-Ames (RSA) Seyfert sample (Maiolino \& Rieke 1995). Fig. 2 (right) shows the average nuclear (typically probing regions of $60 \mathrm{pc}$ in size) spectra for the Seyfert $1 \mathrm{~s}$ and Seyfert 2s galaxies. Clearly, the $11.3 \mu \mathrm{m}$ PAH feature is detected in the average spectrum of both types. We also show the average spectrum of Seyfert galaxies in the RSA sample with deep $10 \mu \mathrm{m}$ silicate features. Such deep features are likely due to dust in the host galaxy and not dust in the torus (Levenson et al. 2007, Alonso-Herrero et al. 2011, Goulding et al. 2012, González-Martín et al. 2013). Nuclear $11.3 \mu \mathrm{m}$ PAH emission is detected in half of the sample with no evidence that the emission is suppressed for more luminous AGN. Esquej et al. (2013) also found a relation between the nuclear SF rate and the black hole accretion rate (see the contribution by P. Esquej in these proceedings) 
that is reproduced by the numerical simulations of Hopkins \& Quataert (2010) for the appropriate physical scales probed by our data. A similar relation is observed when the SF rate is measured on kiloparsec scales (Diamond-Stanic \& Rieke 2012).

\subsection{LLAGN and the origin of the torus}

LLAGN are common in local spirals and ellipticals (Ho 2008). The detection of broad $\mathrm{H} \alpha$ components proves that at least some of them do contain an AGN. Interestingly, at low luminosities $\left(L_{\mathrm{bol}}<10^{42} \mathrm{erg} \mathrm{s}^{-1}\right)$ theoretical models predict that the torus might disappear because accretion can no longer sustain the outflow required for large obscuring columns in the torus (Elitzur \& Shlosman 2006). Moreover, how the properties of LLAGN tori, if they exist, might relate to those in higher luminosity AGN is still not known.

Using sub-arcsecond angular resolution images Mason et al. (2012) found a variety of mid-IR morphologies in a sample of local LLAGN. Sources with higher Eddington ratios tend to show more compact and bright mid-IR sources, whereas LLAGN with low Eddington ratios appear more diffuse and extended in the mid-IR. It is indeed in the former type of objects in which the torus is expected to be detectable in our CC survey. Furthermore, from the modelling of multi-wavelength SEDs recently Mason et al. (2013) suggested that dust may account for the nuclear mid-IR emission of many LLAGN. We refer the reader to the contribution by $\mathrm{R}$. Mason in these proceedings for more details.

Based on observations made with the GTC, instaled in the Spanish Observatorio del Roque de los Muchachos of the Instituto de Astrofsica de Canarias, in the island of La Palma. A. A.-H. acknowledges support from the Spanish Plan Nacional through grant AYA2009-05705-E and from the Universidad de Cantabria.

\section{References}

Alonso-Herrero, A. et al. 2011, ApJ, 736, 82

Alonso-Herrero, A. et al. 2013, ApJ, 779, L14

Antonucci, R. 1993, ARA $8 A, 31,473$

Asensio Ramos, A. \& Ramos Almeida, C. 2009, ApJ, 696, 2075

Diamond-Stanic, A. M. \& Rieke, G. H. 2010, ApJ, 724, 140

Diamond-Stanic, A. M. \& Rieke, G. H. 2012, ApJ, 746, 168

Elitzur, M. \& Shlosman, I. 2006, ApJ, 648, L101

Esquej, P. et al. 2013, ApJ, in press (arXiv:1311.0703)

González-Martín, O. et al. 2013, A\&3A, 553, A35

Goulding, A. D. et al. 2012, ApJ, 755, 5

Ho, L. C. 2008, ARA\&A A, 46, 475

Hopkins, P. F. \& Quataert, E. 2010, MNRAS, 407, 1529

Levenson, N. A. et al. 2007, ApJ, 645, L45

Maiolino, R. \& Rieke, G. H. 1995, ApJ, 454, 95

Mason, R. E. et al. 2012, AJ, 144, 11

Mason, R. E. et al. 2013, ApJ, 777, 164

Nenkova, M. et al. 2008, ApJ, 685, 160

Packham, C. et al. 2005, ApJ, 618, L17

Packham, C. et al. 2007, ApJ, 661, L29

Ramos Almeida, C. et al. 2009, ApJ, 702, 1127

Ramos Almeida, C. et al. 2011, ApJ, 731, 92

Roche, P. F. et al. 2006, MNRAS, 367, 1689

Telesco, C. M. et al. 2003, SPIE, 4841, 913

Tristram, K. R. W. et al. 2009, A\&A A, 502, 67

Wada, K. \& Norman, C. A. 2002, ApJ, 566, L21 